\title{
Open Data Portal Prototype for the Conservation of Biodiversity of the 'Universidad Nacional de Loja'
}

\section{Prototipo de un Portal de Datos Abiertos para la Conservación de la Biodiversidad de la Universidad Nacional de Loja}

\section{Milton Labanda-Jaramilloํ, Luis Chamba-Eras ${ }^{1}$, Edison Coronel-Romero ${ }^{1}$, Jose-Luis Granda ${ }^{1}$, Angel Marcelo Gutierrez Riofrío², and Lorena Elizabeth Conde Zhingre ${ }^{3,4}$}

${ }^{1}$ Grupo de Investigación en Tecnologías de la Información y Comunicación (GITIC), Carrera de Ingeniería en Sistemas, Facultad de Energía, Universidad Nacional de Loja, Av. Pío Jaramillo Alvarado, Loja, Ecuador

Corresponding Author: Milton Labanda-Jaramillo miltonlab@unl.edu.ec

Received: 4 December 2018 Accepted: 5 December 2018

Published: 27 December 2018

Publishing services provided by Knowledge $\mathrm{E}$

(c) Milton Labanda-Jaramillo et al. This article is distributed under the terms of the Creative Commons Attribution License, which permits unrestricted use and redistribution provided that the original author and source are credited.

Selection and Peer-review under the responsibility of the SIIPRIN-CITEGC Conference Committee

\section{S OPEN ACCESS}

2Jardín Botánico "Reinaldo Espinoza", Facultad Agropecuaria, Universidad Nacional de Loja, Av. Pío Jaramillo Alvarado, Loja, Ecuador

3Universidad Internacional Del Ecuador, Escuela de Informática y Multimedia, Titulación Ingeniería en Tecnologías de la Información, Quito, Ecuador

${ }^{4}$ Instituto Tecnológico Superior Daniel Álvarez Burneo, Carrera de Análisis de Sistemas, Loja, Ecuador

\section{Abstract}

This article describes the possibilities of research and development that exist in the 'Universidad Nacional de Loja' (UNL) in the field of biodiversity through the use and exploitation of information and communication technology (ICT) resources. First, the existing methodologies, processes, and initiatives for the publication of open data was explored. Subsequently, a brief description of the biodiversity conservation centers maintained by the UNL was made, highlighting the IT initiatives that have been carried out. Finally, the results of the proposal include the methodology for the release of open data and the creation of the prototype of the biodiversity open data portal of the UNL.

\section{Resumen}

Este trabajo describe las posibilidades de investigación y desarrollo que existen en la "Universidad Nacional de Loja" (UNL) en el campo de la biodiversidad, por medio del uso y explotación de las Tecnologías de la Información y Comunicación (TIC). En primero lugar, se explora las metodologías existentes, procesos e iniciativas para la publicación de datos abiertos. Seguidamente, se describen brevemente los Centros de Conservación de la Biodiversidad pertenecientes a la UNL, destacando las iniciativas relacionadas con las TIC, que ya han sido desarrolladas. Finalmente, los resultados de la propuesta incluyen una metodología para la liberación de datos abiertos y la creación de un prototipo de portal de datos abierto sobre biodiversidad. 
Keywords: programming, open data, biodiversity, botany, artificial intelligence

Palabras clave: programación, datos abiertos, biodiversidad, botánica, inteligencia artificial

\section{Introducción}

Países como Alemania, Suecia, México y Canadá, están implementando portales de datos abiertos, con base en las Tecnologías de la Información y Comunicación (TIC) de fuente abierta, orientados a la libre publicación de información; así se puede plantear el aprovechamiento del potencial que tienen los datos abiertos y el software libre como herramientas para fortalecer diferentes áreas de conocimiento; y en este sentido el área de la biodiversidad.

La Universidad Nacional de Loja (UNL), en el área de la biodiversidad, ha generado información que permanece cautiva dentro de los precarios sistemas de informáticos asignados a los centros de conservación (Jardín Botánico fundado en 1940). Lo más cercano a información abierta, es un pequeño grupo de páginas web estáticas, dentro del portal institucional de la UNL.

En Ecuador está vigente el Plan Nacional de Gobierno Electrónico, que procura con sus estrategias generar y favorecer ambientes para la liberación de datos que coadyuven a la innovación social, ciudadana y abierta. Por esta razón, se desprende de este plan, la guía de política pública de datos abiertos que persigue la liberación de datos en el marco de la LOTAIP [2]. Así mismo, se propone un conjunto de pasos para proveer de manera pública y ética hacia el mundo la información de las instituciones ecuatorianas: selección de los conjuntos de datos, preparación de los datos y publicación de los datos con una licencia abierta (ver Tabla 1).

Por otro lado, la existencia de la Estrategia Nacional de Biodiversidad [9] impulsa el desarrollo y la implementación de herramientas como el Sistema de Información sobre Biodiversidad (SIB) integradas al Sistema Único de Información Ambiental. Además, la Agenda Nacional de Investigación sobre la Biodiversidad [10] contiene dentro de la meta 1 "Gestión de la información e investigación básica sobre la diversidad biológica en el Ecuador", objetivos muy claros que vinculan de manera directa a la utilización 
TABLA 1: Licencias de datos abiertos.

Nombre
Creative Commons Zero
(CCo)
Creative Commons
Reconocimiento (CC BY)
Creative Commons -
Reconocimiento Compartir
Igual (CC BY-SA)
Public Domain Dedication
and License (PDDL)
Open Data Commons
Attribution License
(ODC-By)
Open Data Commons Open
Database License (ODbL)

Descripción
Ningún tipo de restricción, renunciando el creador
a cualquier derecho sobre la obra, según permita
la ley.
Reconocimiento al trabajo del autor
Reconocimiento al trabajo del autor y bajo las
mismas condiciones.
Permite difundir, reutilizar o adaptar los datos sin
restricción alguna.
Se exige la referencia a la autoría o fuente de los
datos para la reutilización de la información.
Permite la reutilización de los datos siempre que
se reconozca la autoría de la información original

Autor
Creative
Commons
Creative
Commons
Creative
Commons
OKFN (Open
Knowledge
Foundation
Network)
OKFN (Open
Knowledge
Foundation
Network)
OKFN (Open
Knowledge
Foundation
Network)

de las TIC con el fin de potenciar la conservación y la producción basada en la biodiversidad, entre ellos: el objetivo 1.3 "Consolidar la Base Nacional de Datos sobre Biodiversidad (BNDB), integrada al sistema nacional de información de la biodiversidad. Desarrollo de la estructura informática de la BNDB, modelo de gestión y protocolos de registro de datos. Coordinación con entidades generadoras de datos sobre biodiversidad y suscripción de acuerdos para unificar y facilitar el intercambio de información de datos pertinentes para la conservación y utilización sostenible de la diversidad biológica. Depuración, validación de bases de datos de las entidades generadoras e interconexión de nodos territoriales y temáticos con la BNDB" y así mismo en la meta 3, el objetivo 3.1 de la misma agenda [10] menciona "Aplicación de la bioinformática, la biología computacional, la automatización y otras TIC en los procesos de generación, gestión y análisis de datos biológicos para apoyar el uso sostenible y la conservación de la biodiversidad en taxones y ecosistemas clave".

Como se menciona en [7], en América Latina existe carencia en recursos humanos en TIC aplicadas al dominio de conservación de la biodiversidad, producto de la poca importancia que brindan las autoridades, docentes e investigadores a los centros y espacios relacionados con la conservación de la biodiversidad. Quizá por desconocimiento de las posibilidades de potenciarlos a través del uso de las TIC o debido quizá a las problemáticas internas propias de su gestión administrativa y/o académica. 
El jardín botánico "Reinaldo Espinosa" posee un blog que es gestionado por el personal técnico del mismo, y a pesar de que han existido algunas iniciativas e intentos por generar innovación a través del uso de las TIC, considerando el proyecto de fin de carrera denominado "Diseño e implementación del sitio Web para el Jardín Botánico Reinaldo Espinosa de la Universidad Nacional de Loja con el recorrido virtual del laberinto" [8] no ha existido implementación alguna de proyectos exitosos relacionados con las TIC. La implementación real del proyecto mencionado no se pudo ejecutar, por lo tanto, la base de datos con la información levantada de las especies del jardín se descontinuó totalmente, haciendo que los técnicos del jardín botánico opten por generar una nueva base de datos con el software "Filemaker", encontrándose actualmente en construcción.

Dentro del portal institucional de la UNL existe reducida información del jardín botánico "Reinaldo Espinosa" y de la misma forma para el herbario "Reinaldo Espinosa".

El objetivo principal de este trabajo es presentar una propuesta metodológica para publicar la información sobre la biodiversidad del jardín botánico "Reinaldo Espinosa" mediante un portal de datos abiertos de biodiversidad. Este trabajo se organiza de la siguiente forma: la sección 1 presenta los procesos de publicación de datos abiertos, el marco jurídico en Ecuador, biodiversidad y las TIC en la UNL. En la sección 2 se detalla brevemente la contextualización sobre el objeto de estudio. La sección 3 detalla la metodología utilizada en el contexto de estudio. La sección 4 muestra los resultados preliminares. Finalmente, en la sección 5 se describen las conclusiones y las líneas futuras.

\section{Datos abiertos y Biodiversidad}

Al entender como biodiversidad todo el conjunto de formas de vida diferentes que pueblan el planeta, hoy en día los jardines botánicos, por ejemplo, del latín "hortus botanicus", se erigen como instituciones habilitadas por organismos públicos, privados o asociativos (en ocasiones la gestión es mixta) cuyo objetivo es el estudio, la conservación y divulgación de la diversidad vegetal. Se caracterizan por exhibir colecciones científicas de plantas vivas, que se cultivan para conseguir alguno de estos objetivos: su conservación, investigación, divulgación y enseñanza.

A su vez los herbarios, dentro de un jardín botánico, tienen una función científica, pero, sobre todo, tienen el cometido de conservar la memoria, cosa absolutamente indispensable. Esta memoria permitirá, con el transcurso de los años, conocer aquellas plantas que un día estuvieron presentes en el cultivo de los jardines. Los herbarios 
son una fuente de información acerca de las plantas y del medio en el que habitan y suponen en sí mismos un registro permanente de la biodiversidad.

La UNL posee centros de conservación ex-situ de la flora nativa y endémica de la región, entre ellos resalta el jardín botánico "Reinaldo Espinoza" que alberga dentro de su estructura a un herbario y al parque universitario descritos a detalle en [6], constituyéndose desde hace casi 70 años en el nicho apropiado para impulsar la investigación y la academia relacionadas con la biodiversidad y el medio ambiente.

\section{Metodología}

La Asociación Civil Red-Ciudadana en [1] presenta una metodología utilizada en proyectos enfocados a la apertura de datos de gobiernos como el de Guatemala: diagnóstico, la planificación, la publicación, la promoción, socialización y la validación. En Ecuador, según la "Guía de Política Pública de Datos Abiertos de la Secretaría de la Administración Pública de la República del Ecuador" [2] propone para la apertura de datos: selección del conjunto de datos, preparación de datos, publicación de datos (selección de licencias de uso, inclusión de metadatos, publicación de los datos, ubicación del enlace de los datos abiertos en los portales institucionales), interacción ciudadana, comentarios y solicitud de data sets. En la Universidad Central del Ecuador [3] el proyecto "Portal de datos abiertos de la Universidad Central del Ecuador", dentro de la metodología, se menciona la identificación de riesgos según el Procesos de desarrollo Ágil Unificado (AUP) y que se basó en la Ley Orgánica de Transparencia y Acceso a la Información Pública (LOTAIP) durante la identificación, recolección, publicación y actualización de datos abiertos. El gobierno español en [4] establece: selección de los datos que se van a publicar, establecer las licencias para facilitar la reutilización, preparación de los datos, publicación de los datos, evaluación de uso, mantenimiento de los datos. Por su parte el "Departamento de Asuntos Económicos y Sociales de las Naciones Unidas" [5] dicta los siguientes pasos: entender la oferta y demanda, priorizar los lanzamientos de datos, publicar datos bajo los principios de datos abiertos, promover y apoyar la reutilización, institucionalizar una política de datos abiertos, hacer las guías de datos abiertos parte del estándar de manejo de datos, mejorar las capacidades analíticas de datos. El Ministerio de TIC del gobierno colombiano en su "Guía de Datos Abiertos en Colombia" [11] engloba en tres grandes fases todo el proceso de publicación: documentación, estructuración y carga de datos. 
Conociendo las experiencias anteriormente descritas, primeramente, se realizó una investigación exploratoria, relacionada a la búsqueda de portales o sitios Web relacionados con biodiversidad en la Web, usando como descriptores las cadenas "portal biodiversidad datos abiertos" en Google Académico, con el fin de encontrar sitios Web que expongan información como datos abiertos relacionadas con biodiversidad con los cuales a futuro se podría interactuar con el fin de reutilizarla y complementar los conjuntos de datos que se generen in situ (ver Tabla 2). Posteriormente, se realizó una observación in situ al jardín botánico "Reinaldo Espinoza" y se identificó los avances y proyectos de TIC que se han implementado, así como también las proyecciones que se tiene con la ayuda de las TIC. Finalmente, existen muchas universidades en Latinoamérica que poseen jardines botánicos, se hizo por tanto una revisión de diez portales de universidades junto con la cantidad de datos que exponen, identificando que la mayoría tiene un portal de sus jardines botánicos o herbarios, pero ninguna tiene una iniciativa de datos abiertos implementada y evidenciada públicamente (ver Tabla 3).

TABLA 2: Portales que mantienen datos abiertos de biodiversidad.

\begin{tabular}{|c|c|c|}
\hline Nombre & Acrónimo & Enlace \\
\hline $\begin{array}{l}\text { Comisión Nacional para el } \\
\text { Conocimiento y Uso de la } \\
\text { Biodiversidad }\end{array}$ & CONABIO & https://www.gob.mx/conabio \\
\hline $\begin{array}{l}\text { Global Biodiversity Information } \\
\text { Facility }\end{array}$ & GBIF & https://www.gbif.org/ \\
\hline $\begin{array}{l}\text { Ministerio de Agricultura y Pesca, } \\
\text { Alimentación y Medio Ambiente de } \\
\text { Nombre }\end{array}$ & MAPAMA & http://www.mapama.gob.es/es/ \\
\hline Belgian biodiversity data porta & Biodiversity.be & http://data.biodiversity.be/ \\
\hline $\begin{array}{l}\text { Sistema de Información de la } \\
\text { Naturaleza Europea }\end{array}$ & EUNIS & http://eunis.eea.europa.eu/ \\
\hline $\begin{array}{l}\text { Sistema Nacional de Datos } \\
\text { Biológicos de Argentina }\end{array}$ & SNDB & http://datos.sndb.mincyt.gob.ar \\
\hline Atlas de Biodiversidad & CRBio & http://www.crbio.cr \\
\hline Portal da Biodiversidade & PortalBio & $\begin{array}{l}\text { https://portaldabiodiversidade. } \\
\text { icmbio.gov.br/portal/ }\end{array}$ \\
\hline BIOWEB & BIOWEB & https://bioweb.bio/ \\
\hline $\begin{array}{l}\text { Ministerio del Poder Popular para el } \\
\text { Ecosocialismo y Aguas }\end{array}$ & & $\begin{array}{l}\text { http://diversidadbiologica.minamb. } \\
\text { gob.ve/plataforma }\end{array}$ \\
\hline
\end{tabular}

De la revisión presentada, se describen los resultados preliminares para publicar como datos abiertos la información sobre la biodiversidad del jardín botánico "Reinaldo Espinosa", la misma que se organizaron en siete fases: 
TABLA 3: Portales de jardines botánicos y/o herbarios de universidades latinoamericanas.

\begin{tabular}{|c|c|c|c|}
\hline Universidad & País / Ciudad & Enlace & Datos \\
\hline $\begin{array}{l}\text { Universidad Federal Santa } \\
\text { María }\end{array}$ & Brasil / Rio Grande do Sul & http://w3.ufsm.br/jbsm/ & 1830 \\
\hline Universidad de Puerto Rico & Puerto Rico / San José & $\begin{array}{l}\text { http: } \\
\text { //www.upr.edu/jardin-botanico/ }\end{array}$ & 84 \\
\hline $\begin{array}{l}\text { Universidad Tecnológica de } \\
\text { Pereira }\end{array}$ & Colombia / Регеiгa & https://www.utp.edu.co/jardin/ & 1990 \\
\hline Universidad de Costa Rica & Costa Rica / Cartago & http://www.jbl.ucr.ac.cr/ & - \\
\hline Universidad de Caldas & Colombia / Caldas & $\begin{array}{l}\text { http://www.ucaldas.edu.co/ } \\
\text { portal/jardi\%C2\%ADn-botanico/ }\end{array}$ & 167 \\
\hline $\begin{array}{l}\text { Benemérita Universidad } \\
\text { Autónoma de Puebla }\end{array}$ & México / Puebla & $\begin{array}{l}\text { http: } \\
\text { //www.jardinbotanico.buap.mx/ }\end{array}$ & 418 \\
\hline $\begin{array}{l}\text { Universidad Austral de } \\
\text { Chile }\end{array}$ & Chile / Los Ríos & http://jardinbotanicouach.cl/ & 1060 \\
\hline Universidad de los Andes & Venezuela / Mérida & $\begin{array}{l}\text { http://www.ciens.ula.ve/ } \\
\text { jardinbotanico/ }\end{array}$ & 1170 \\
\hline $\begin{array}{l}\text { Universidad Nacional } \\
\text { Agraria de la Selva }\end{array}$ & Perú / Tingo María & $\begin{array}{l}\text { https://www.unas.edu.pe/web/ } \\
\text { content/jard\%C3\%ADn-botanico }\end{array}$ & 3 \\
\hline
\end{tabular}

Fase 1: conformación el comité de datos abiertos, según lo estipula la "Guía de Política Pública de Datos Abiertos de la Secretaría de la Administración Pública de la República del Ecuador" [2].

Fase 2: levantamiento de la información de biodiversidad del jardín botánico "Reinaldo Espinoza".

Fase 3: migración de la información a formatos de datos abiertos de nivel 3: XML, CSV y JSON, de acuerdo a los posibles usuarios potenciales de la información, considerando los metadatos propuestos en la "Guía de aplicación de la Norma Técnica de Interoperabilidad de reutilización de recursos de información del sector público de España" [11] y $[4]$.

Fase 4: publicación de datos de biodiversidad del jardín botánico "Reinaldo Espinoza" a través del prototipo de portal de datos abiertos versión alfa (Dirección Web del prototipo: http://46.101.25.247:8000/UnIEcBiodiversidad) (ver Figura 1).

Fase 5: implementación el Sistema de Información de Biodiversidad de la UNL el cual deberá obligatoriamente usar estándares abiertos de información de biodiversidad, tales como el ITF (Internacional Transfer Format for Botanical Garden Data) y el HISPID (Herbarium Information Standard and Protocol for Interchange of Data) en sus versiones más actuales. 
Fase 6: implementación de una interfaz de programación de aplicaciones (API) que soporte el protocolo de intercambio de información REST para proveer información principalmente a los sistemas e iniciativas a nivel nacional tales como el Portal de Biodiversidad Ecuatoriana (bioweb), el Sistema Único de Información Ambiental y la Base Nacional de Datos sobre Biodiversidad (BNDB) así como también permitir la interacción con sistema mundialmente reconocidos como la plataforma GBIF.

Fase 7: publicación del sistema de información de biodiversidad en el portal Web de la UNL.

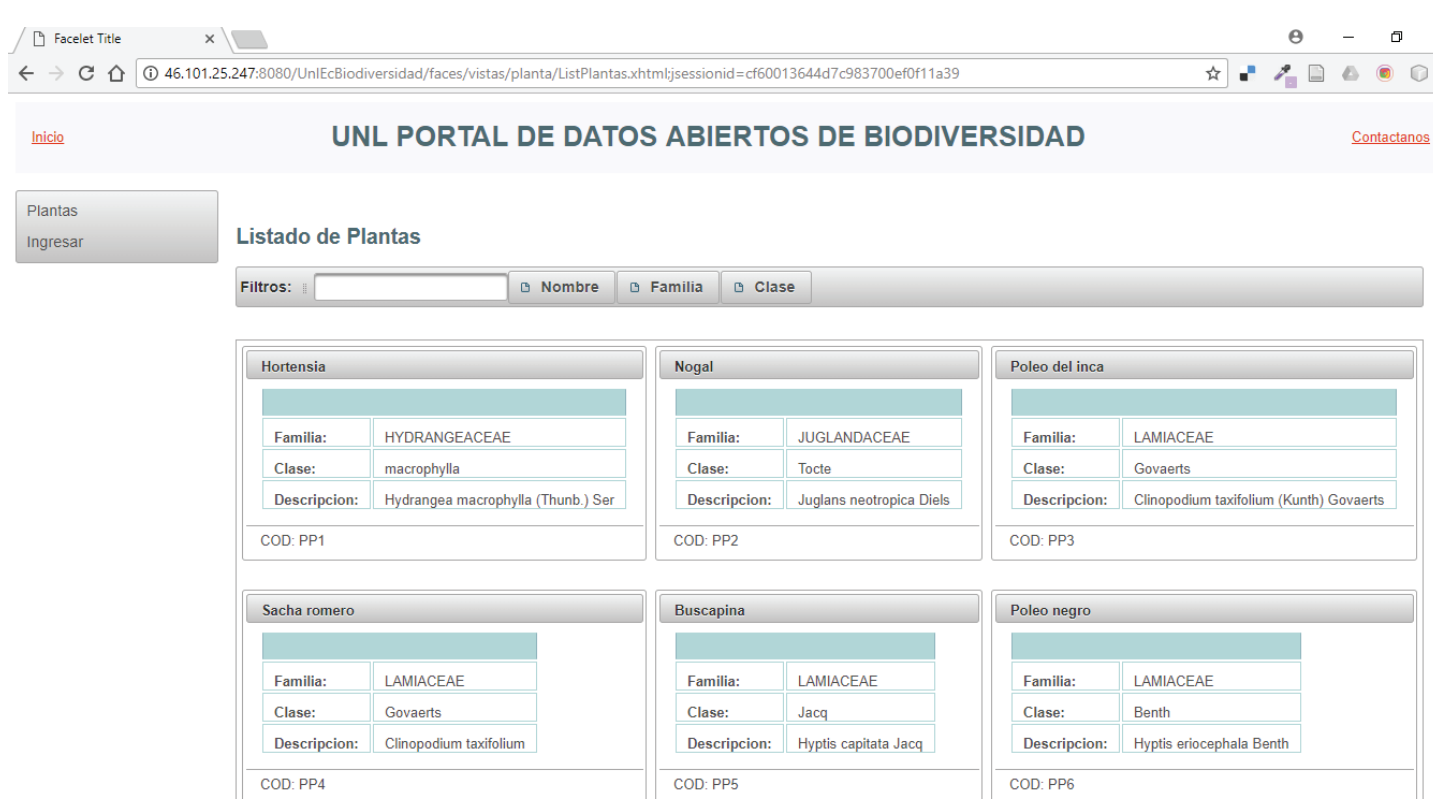

Figura 1: Buscador de plantas del prototipo de portal de datos abiertos de biodiversidad.

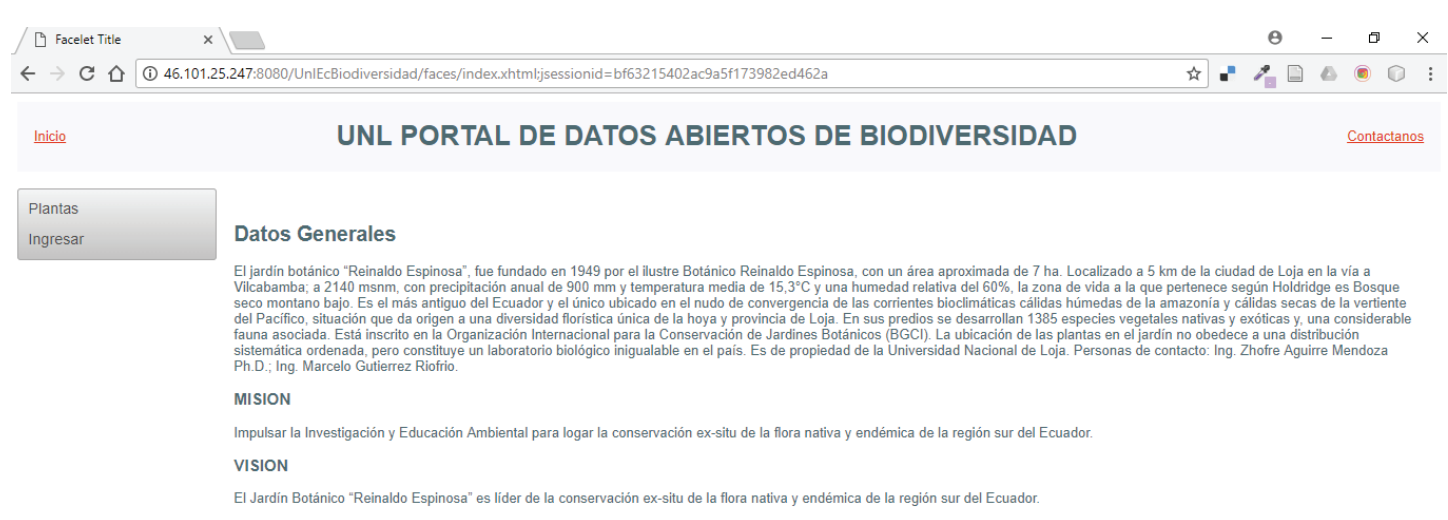

FIgura 2: Página de inicio del prototipo de datos abiertos de biodiversidad. 


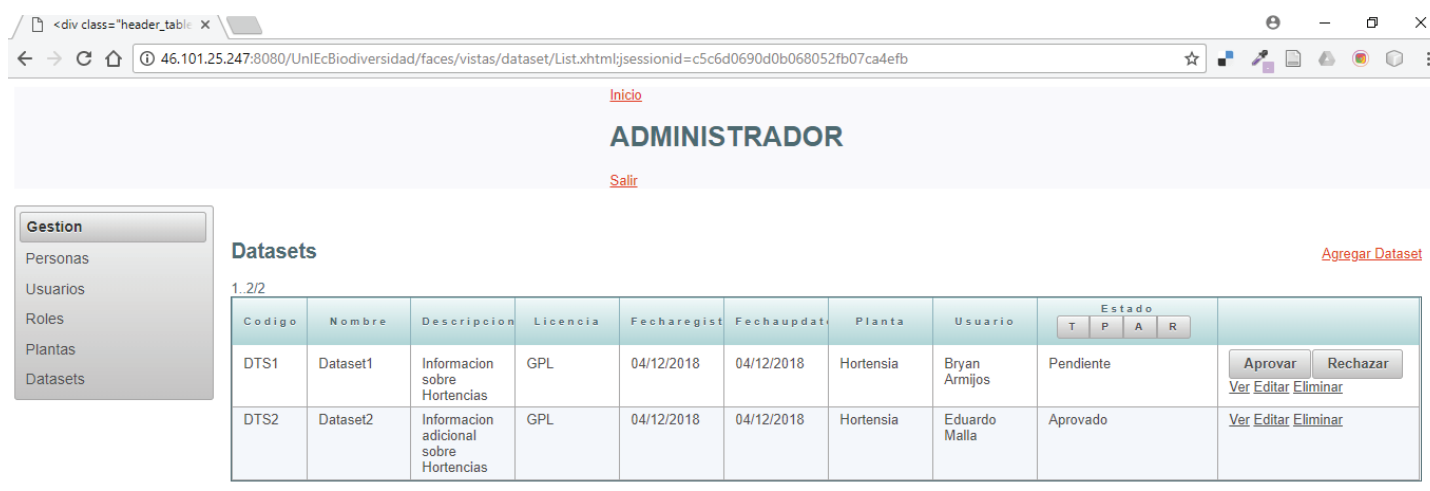

Figura 3: Gestión de conjuntos de datos del prototipo de datos abiertos de biodiversidad.

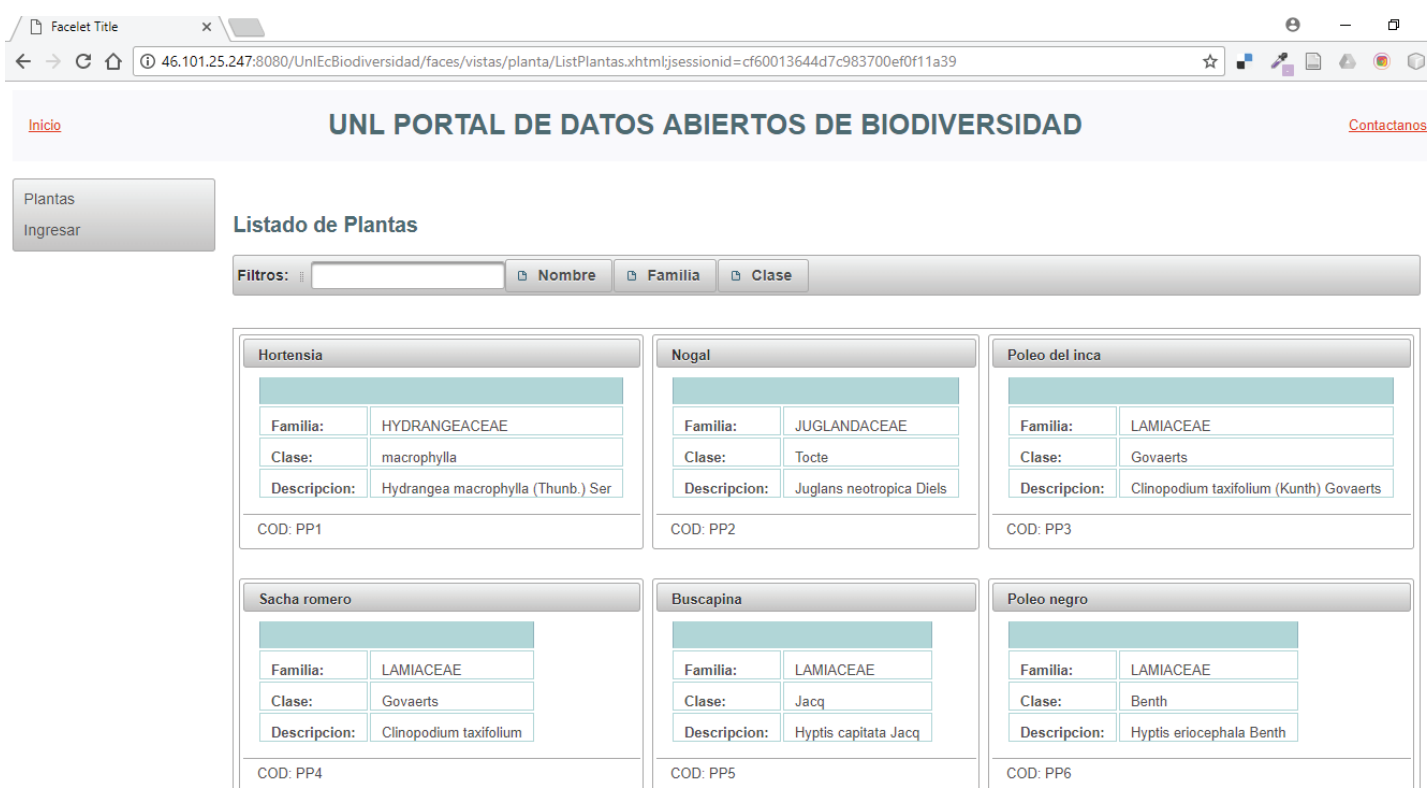

Figura 4: Buscador de plantas del portal de datos abiertos de biodiversidad.

\section{Resultados preliminares}

De acuerdo a lo descrito en la Tabla 2, existen a nivel mundial iniciativas y organizaciones que han implementado portales y/o sistemas de información relacionados con la biodiversidad, cuyo objetivo, por lo general, es difundir, incrementar y compartir el conocimiento de países, jardines botánicos, herbarios y otras formas de conservación de la biodiversidad, algunas de ellas a través de buscadores simples en línea y otras hasta a través del aprovisionamiento de interfaces y APIs de programación que pueden ser usadas o consumidas desde distintas plataformas informáticas. Las Universidades que poseen centros de conservación de biodiversidad están obligadas, se puede decir, tanto por su carácter primario de generadoras de conocimiento y por su responsabilidad de conservación del mismo, a proveerlo a la sociedad; lo cual se contrasta con lo descrito en la Tabla 3, en donde diversas universidades de Colombia, Brasil y Chile son 
las que mayor cantidad de resultados arrojan al realizar búsquedas en sus portales. El prototipo versión alfa, planteado para la implementación del Portal de Datos Abiertos, actualmente contiene entre las funcionalidades principales: la búsqueda de plantas, la búsqueda y gestión de conjuntos (datasets) de datos abiertos, que servirán de base para la implementación futura de las APIs, interfaces y servicios Web que pondrán a disposición del público los datos de la biodiversidad que la UNL alberga y conserva dentro jardín botánico, herbario y parque universitario, y posiblemente a mediano plazo, la biodiversidad del centro Binacional "Zapotepamba" y la estación experimental el "Padmi".

\section{Conclusiones y líneas futuras}

Existen algunos elementos de base ya existentes que pueden repotenciarse con el fin de generar proyectos que vinculen la informática para la biodiversidad dentro de la UNL, a través de proyectos de investigación, trabajos de titulación, trabajos de fin de máster, pasantías entre otros.

La propuesta metodológica de publicación de datos abiertos a cerca de la biodiversidad que existe en el jardín botánico de la UNL está enmarcada dentro del marco jurídico del estado ecuatoriano.

Una de las limitantes que se identifica en la investigación y que se debe considerar para las propuestas futuras que involucren las TIC y biodiversidad, consiste en la carencia de un levantamiento/inventario de todas las especies y especímenes existentes dentro del jardín botánico, herbario y parque universitario de la UNL.

La implementación del portal o sistema de información mediante el cual se pondrá a disposición los datos abiertos de la biodiversidad que posee el jardín botánico de la UNL y sus secciones, abrirá las expectativas para que puedan ser utilizados, reutilizados e integrados en las iniciativas nacionales e internacionales relacionadas con biodiversidad y medio ambiente.

Como líneas futuras, se plantea la aplicación de técnicas de Big Data sobre los datos de biodiversidad de la UNL integrados a su vez con los datos de los portales y sistemas de información a nivel nacional, aplicación de Web Semántica sobre el acceso y consumo de información del portal de datos abiertos de biodiversidad, desarrollo de aplicaciones móviles para la captura de información de biodiversidad a través de la utilización realidad aumentada, uso del portal de datos abiertos de biodiversidad en la enseñanza de asignaturas relacionadas. 


\section{Agradecimientos}

Este trabajo forma parte de las actividades del Grupo de Investigación GITIC, agradecemos a los estudiantes de la asignatura de programación del tercer ciclo de la Carrera de Ingeniería en Sistemas, de la Facultad de Energía de la Universidad Nacional de Loja por su colaboración en el presente trabajo. De igual manera se reconoce la colaboración del Ingeniero Bryan Armijos por su apoyo en el desarrollo del prototipo del portal de datos abiertos de biodiversidad para la UNL.

\section{Referencias}

[1] J. Herrera, "Metodología de Apertura de Datos," 2010.

[2] Secretaría Nacional de la Administración Pública, Guía de política pública de en datos abiertos -GPP-DA-v01-2014. 2014, P. 11.

[3] E. Robert, H. Maribel, and R. Maritza, "Portal de Datos Abiertos de la Universidad Central del Ecuador," p. 111, 2013.

[4] E. y T. Ministerio de Industria, "Cómo publicar datos abiertos de manera rápida ápida y sencilla (con ckan)." pp. 1-20, 2015.

[5] UN, "La forma correcta de publicar Datos Abiertos," no. May. Departamento de Asuntos Sociales y Económicos, 2015.

[6] Z. Aguirre, M. Gutierrez, T. Gaona, and N. Jaramillo, "Escenarios para la enseñanza y la valoración de la Biodiversidad en la Región Sur del Ecuador," Bosques Latid. Cero, vol. 6, no. 2, pp. 73-87, 2016.

[7] C. Garita and E. Mata, "Desarrollando Capacidades en Informática para la Biodiversidad en América Latina," no. November, 2007.

[8] J. Jaya and C. Pauta, "Diseño e implementación del sitio Web para el jardín botánico de la Universidad Nacional de Loja con el recorrido virtual del laberinto," 2010.

[9] M. del Ambiente, Estrategia Nacional de Biodiversidad 2015-2030 - Calidad Web.pdf. 2017, P. 225.

[10] INABIO, "Agenda nacional sobre la biodiversidad." SENESCYT, p. 11, 2018.

[11] Ministerio de Hacienda y Administraciones Públicas, "Guía de aplicación de la Norma Técnica de Interoperabilidad de Reutilización de recursos de información." pp. 7781, 2016.

[12] MINTIC, "Guía de datos abiertos en Colombia". Versión 3. 2016. 